Originalien

Z. Epileptol. 2021 · 34:324-330 https://doi.org/10.1007/s10309-021-00415-2 Angenommen: 20. April 2021

Online publiziert: 8. Juni 2021

(c) Der/die Autor(en) 2021
Therapierefraktäre fokale Epilepsien haben im Allgemeinen eine hohe Krankheitslast, weshalb ein epilepsiechirurgischer Eingriff in diesen Fällen oftmals die Therapie der 1. Wahl ist, um Krankheitsfolgen zu mildern und die Lebensqualität zu verbessern $[1,2]$. Insbesondere bei jungen Patienten kann ein hypothalamisches Hamartom $(\mathrm{HH})$ die Ursache einer oftmals therapierefraktären Epilepsie sein [3]. Begleitend sind $\mathrm{HH}$ mit einem höheren Risiko für psychobehaviorale Auffälligkeiten (in bis zu $60 \%$ mit Aggressivität, Hyperaktivität, generalisierter Angststörung oder Phobien) und endokrinologische Störungen wie einer Pubertas praecox verbunden $[3,4]$. $\mathrm{Zu}$ der klassischen Anfallssemiologie gehören gelastische (Lach-) oder dakrystische Anfälle (Weinanfälle). Allerdings können im Verlauf mit Ausweitung des epileptischen Netzwerkes auch dialeptische, tonisch-klonische und Sturzanfälle bis hin zu schweren kognitiven Störungen im Sinne einer epileptischen Enzephalopathie beobachtet werden [3]. Therapierefraktäre Verläufe können dabei einen

Konstantin Kohlhase ${ }^{1,2} \cdot$ Adam Strzelczyk $^{1,2} \cdot$ Luciana Porto $^{3} \cdot$ Achim Beißel $^{4}$. Daniel J. Curry ${ }^{5}$. Felix Rosenow ${ }^{1,2}$. Susanne Schubert-Bast ${ }^{1,2}$

'Klinik für Neurologie und Epilepsiezentrum Frankfurt Rhein-Main, Goethe-Universität Frankfurt, Frankfurt am Main, Deutschland

${ }^{2}$ LOEWE Center for Personalized and Translational Epilepsy Research (CePTER), Goethe-Universität Frankfurt, Frankfurt am Main, Deutschland

${ }^{3}$ Institut für Neuroradiologie des Zentrums der Radiologie, Goethe Universität Frankfurt, Frankfurt am Main, Deutschland

${ }^{4}$ Stationäre Versorgung, BIG direkt gesund, Dortmund, Deutschland

${ }^{5}$ Department of Pediatric Neurosurgery, Texas Children's Hospital, Baylor College of Medicine, Houston, USA

\title{
MR-gestützte stereotaktische Laserthermoablation eines hypothalamischen Hamartoms bei einer jungen Epilepsie- patientin: klinischer Verlauf und Kostenanalyse
}

weitreichenden Einfluss auf die Gesundheit und Lebensqualität des betroffenen Kindes als auch die psychosozialen Strukturen von Familie und Umfeld haben. Damit einhergehend, können hohe direkte, indirekt und intangible Kosten entstehen $[5,6]$. Zwar bietet eine epilepsiechirurgische Behandlung eine realistische Chance auf eine dauerhafte Anfallsfreiheit, birgt aufgrund der zentralen Lage des $\mathrm{HH}$ allerdings ein relevantes perioperatives Komplikationsrisiko, welches bei einer mikrochirurgischen Resektion zwischen 8 und $50 \%$ liegt [7]. $\mathrm{Zu}$ den Komplikationen zählen Thalamusinfarkte, Gesichtsfelddefekte, endokrinologische Störungen und Gedächtnisprobleme [8]. Eine vergleichsweise schonende und dennoch hocheffektive Alternative stellen minimal-invasive Verfahren wie die MR-gestützte stereotaktische Laserthermoablation (SLTA) dar $[9,10]$.

Im Folgenden berichten wir über eine junge Patientin im mittleren Kindesalter mit einer medikamentenrefraktären Epilepsie und einer Pubertas praecox bei $\mathrm{HH}$ und der Abwägung zwischen einer offe- nen operativen Therapie und einer SLTA. Darüber hinaus stellen wir den klinischen Verlauf nach dem Eingriff und eine gesundheitsökonomische Analyse dar.

\section{Methoden}

Die direkten Kosten wurden aus der Perspektive der Gesetzlichen Krankenversicherung (GKV) für die Versicherungsjahre 2017 bis 2020 in Euro berechnet und nach Kostenkomponenten (ambulante Behandlung, stationäre Behandlung, Medikamente nach Indikationsgebiet und Sonstiges) aufgeteilt [11]. Die Datengrundlage bildeten die abgerechneten Kosten der Krankenkasse im Eurowert $(€)$ des jeweiligen Versicherungsjahres, eine Anpassung an die Inflation erfolgte nicht. Eine Abschätzung der indirekten Kosten, die bei den Eltern entstanden, wurde nicht durchgeführt. 

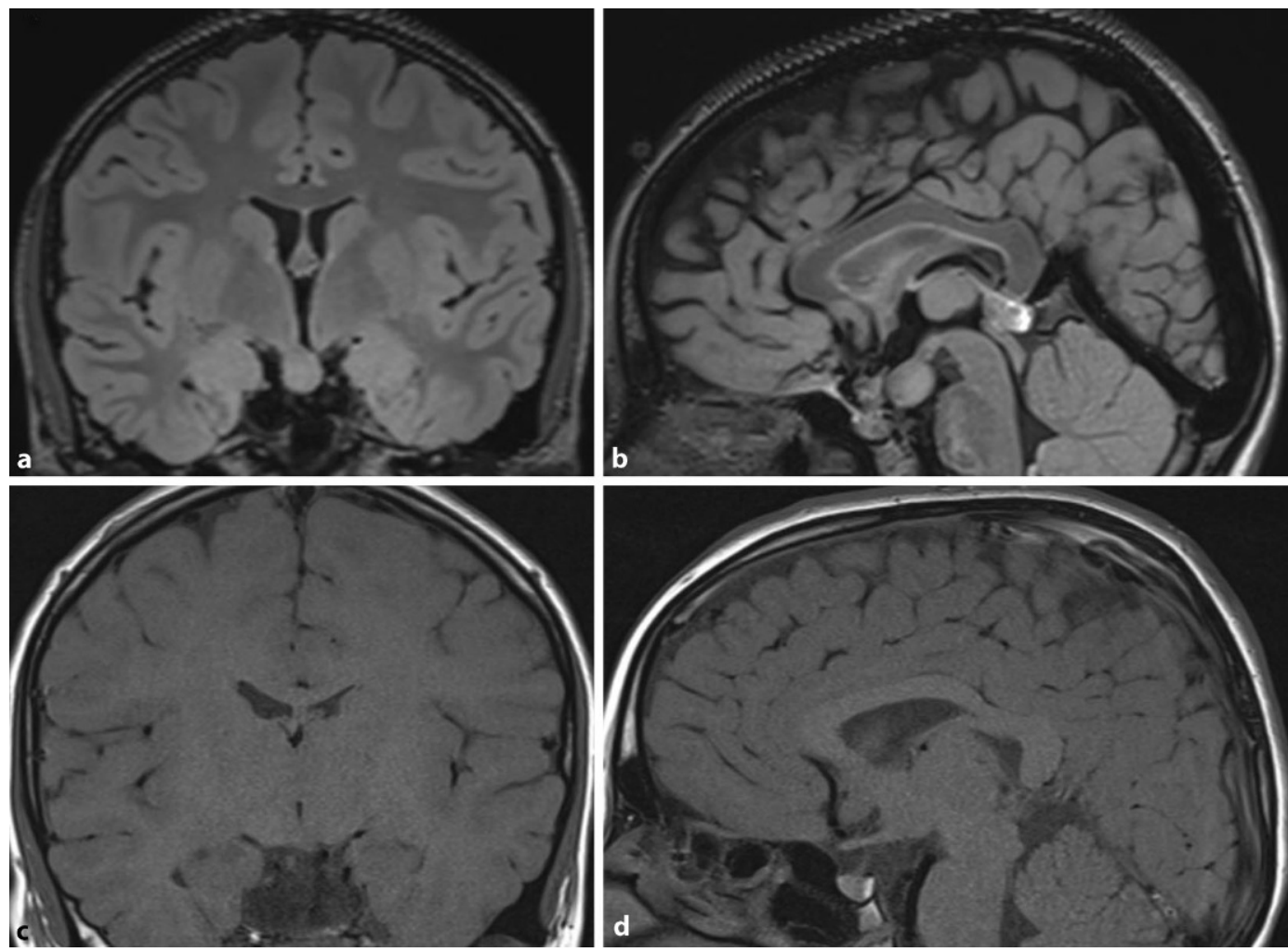

Abb. $1 \varangle$ MRT des hypothalamischen Hamartoms prä- $(\mathbf{a}, \mathbf{b})$ und postinterventionell (c, d). Präablative Darstellung von 10/2018 in T2-Dark-Fluid-Sequenz koronar (a) und sagittal (b) mit einer initialen Größe von $10 \times 9,5 \times 12 \mathrm{~mm}$ (Breite $\times$ Höhe $\times$ Tiefe). Postablative Darstellung des Hamartoms von 05/2020 in T1-Sequenz nativ koronar (c) und sagittal (d) mit noch verbliebenem Hamartomrest $(2 \times 3 \times 7 \mathrm{~mm})$ am Tuber cinereum rechts

\section{Ergebnisse}

\section{Fallbericht}

Wir berichten über ein Mädchen mit Nachweis eines $\mathrm{HH}$ im Bereich des Tuber cinereum (Delalande Grad I) ([12]; - Abb. 1a, b). Dieses wurde bereits im 4. Lebensjahr im Rahmen der Abklärung einer Pubertas praecox festgestellt, ein erster epileptischer Anfall wurde im Alter von 6 Jahren beobachtet. Semiologisch handelte es sich um gelastische und dialeptische Anfälle, welche mit einem tonischen Anfall des linken Gesichts und linken Arms einhergehen konnten. Insbesondere die dialeptischen Anfälle traten bereits zu Beginn der Erkrankung bis zu mehrfach täglich auf und sprachen auf eine antikonvulsive Medikation nur gering an. Eine Therapie mit Oxcarbazepin (bis $900 \mathrm{mg} / \mathrm{Tag}=\mathrm{ca} .30 \mathrm{mg} / \mathrm{kg} / \mathrm{Tag}$ ), eine Kombinationstherapie mit Brivaracetam (bis $100 \mathrm{mg} / \mathrm{Tag}$ ) und eine Monotherapie mit Lacosamid $(200 \mathrm{mg} / \mathrm{Tag}$ =ca. $7 \mathrm{mg} / \mathrm{kg} / \mathrm{Tag}$ ) blieben erfolglos, sodass die Diagnose einer medikamentenrefraktären strukturellen Epilepsie gestellt wurde. Im Verlauf kam es zu einer Zunahme der Anfallsfrequenz mit mehrmals täglich auftretenden astatischen Anfällen. Zusätzlich litt die Patientin unter vermehrter Müdigkeit, deutlichen Konzentrationsschwierigkeiten und psychiatrischen Auffälligkeiten wie einer Angststörung mit aggressiven Durchbrüchen. Die Erkrankung wirkte sich negativ auf die Schulleistung aus und führte $\mathrm{zu}$ einer zunehmenden sozialen Isolation. Aufgrund der täglichen Anfälle wurden die Eltern wiederholt aufgefordert, das Kind von der Schule abzuholen, da diese mit der Anfallssituation überfordert war. In der neuropsychologischen Testung zeigte sich eine leichte kognitive Störung mit wechselnden Schwächen im Bereich der visuokonstruktiven Verarbeitung sowie des Langzeitgedächtnisses. Aufgrund der Beschwerden wurden durch die betreuenden Ärztinnen und Ärzte eine Integrationsassistenz, ein Fahrdienst und die Beschaffung eines Rollstuhls beantragt.

Angesichts des progredienten Verlaufs erfolgte ein stationäres prächirurgisches Video-EEG-Monitoring, währenddessen mehrere habituelle Anfälle mit einer Anfallsursprungszone frontozentral rechts aufgezeichnet werden konnten [13]. In der interdisziplinären Fallkonferenz (unter Beteiligung von Epileptologie, Neuroradiologie, Neurochirurgie, Neuropsychologie, Nuklearmedizin und Strahlentherapie) wurde die Indikation zur epilepsiechirurgischen Versorgung des $\mathrm{HH}$ gestellt. Angesichts der hohen Komplikationsrate einer offenen Resektion wurde beschlossen, die Krankenkasse um Kostenübernahme für eine MR-gestützte SLTA in Houston, Texas, USA, zu bitten [8]. Diese erfolgte nach Bewilligung der Kostenübernahme durch die BIG-Krankenkasse Anfang November 2018 im Alter von 8 Jahren am Texas Children's Hospital des Baylor College of Medicine in Houston. Zur besseren Visualisierung der epileptogenen Läsion und des epileptogenen Netzwerkes wurde eine funktionelle MRT mit Bestimmung der „resting state connectivity“ (rs-fMRI) im Rahmen einer klinischen Studie ergänzt [14]. Zur Ablation wurden zwei $3 \mathrm{~mm}$ große Bohrlöcher gesetzt, über welche jeweils ein Laserkatheter durch den rechten Frontallappen bis zum $\mathrm{HH}$ geführt wurde (- Abb. 2a). Die Ablation verlief komplikationslos, zur Ödemprophylaxe wurden über 10 Tage Kortikoide (Dexamethason 
Z. Epileptol. 2021·34:324-330 https://doi.org/10.1007/s10309-021-00415-2

(c) Der/die Autor(en) 2021

K. Kohlhase · A. Strzelczyk · L. Porto · A. Beißel · D. J. Curry · F. Rosenow · S. Schubert-Bast

\section{MR-gestützte stereotaktische Laserthermoablation eines hypothalamischen Hamartoms bei einer jungen Epilepsiepatientin: klinischer Verlauf und Kostenanalyse}

\section{Zusammenfassung}

Einleitung. Die stereotaktische Laserthermoablation (SLTA) stellt eine minimal-invasive Behandlung für therapierefraktäre Epilepsien auf dem Boden eines hypothalamischen Hamartoms $(\mathrm{HH})$ dar. Durch die weitreichenden Folgen einer therapierefraktären Epilepsie können hohe direkte Kosten entstehen, die durch eine zu erzielende Anfallsfreiheit gesenkt werden können.

Methoden. Anhand einer Patientin mit einem $\mathrm{HH}$ sollen die Auswirkungen einer solchen Erkrankung beleuchtet und der Krankheitsverlauf nach erfolgter SLTA dargestellt werden. Zur Beurteilung der Kosteneffizienz der SLTA wurden die direkten Kosten, basierend auf den Krankenversicherungsdaten der Patientin, über die Versicherungsjahre 2017 bis 2020 analysiert.
Ergebnisse. Bei der Patientin bestand eine hochaktive, medikamentenrefraktäre Epilepsie mit erhöhtem Verletzungsrisiko und zunehmender Verschlechterung der schulischen Leistung und der psychischen Verfassung. Begleitend bestand durch das HH eine Pubertas praecox. Nach SLTA entwickelte die Patientin mit einem Followup von 26 Monaten eine vollständige Anfallsfreiheit sowie eine endokrinologische Stabilisierung, sodass die antikonvulsive als auch die hormonelle Medikation im Verlauf beendet werden konnten. Relevante persistierende Komplikationen wurden nicht beobachtet. Die direkten jährlichen Kosten (stationär [ausschließlich der SLTA selbst]/ambulant/Medikamente) reduzierten sich von $€ 6603$ in 2017 und $€ 12.903$ in
2018 auf $€ 3609$ in 2019 und zuletzt $€ 617$ in 2020, was einer Reduktion von bis zu $95 \%$ (2018 gegenüber 2020) entsprach. Zusätzlich konnten die Kosten einer geplanten Integrationsassistenz von schätzungsweise $€$ 18.000/Jahr eingespart werden.

Schlussfolgerung. Die SLTA stellt eine effektive und risikoarme Behandlung von $\mathrm{HH}$ dar und führt bereits nach 2 Jahren zu einer relevanten Einsparung der direkten Kosten, was bei der Kosten-Nutzen-Abwägung der SLTA einzubeziehen ist.

Schlüsselwörter

Epilepsiechirurgie · Gelastische Anfälle ·

Pädiatrie · Diskonnektion · Minimal-invasiv

\section{MR-guided stereotactic laser thermoablation of a hypothalamic hamartoma in a girl with drug- refractory epilepsy: clinical course and cost analysis}

\section{Abstract}

Introduction. Stereotactic laser thermoablation (SLTA) is a minimally invasive treatment for refractory epilepsy due to hypothalamic hamartoma $(\mathrm{HH})$. Due to the wide-ranging consequences of a refractory epilepsy, high direct costs may be generated, which can be reduced by achieving seizure freedom.

Methods. The case of a girl with $\mathrm{HH}$ and drug-resistant epilepsy is used to illustrate the impact of this disease and to illustrate the course of the disease following SLTA. To assess the cost-effectiveness of SLTA, direct costs were analyzed based on the patient's health insurance data over the insurance years 2017-2020.
Results. The patient had a highly active, medication-refractory epilepsy with increased risk of injury and worsening of academic performance and mental status and a comorbid pubertas praecox due to the $\mathrm{HH}$. After SLTA, the patient developed complete seizure freedom and endocrinological stabilization during a follow-up of 26 months, so that both anticonvulsant and hormonal medication could be discontinued. No relevant persistent complications were observed. Direct annual costs (inpatient [excluding the SLTA procedure]/outpatient/medications) decreased from $€ 6603$ in 2017 and $€ 12,903$ in 2018 to $€ 3609$ in 2019 and $€ 617$ in 2020 , which corresponded to a reduction of $95 \%$ (2018 vs. 2020). In addition, an estimated $€ 18,000$ /year could be saved by avoiding a planned integration assistance.

Conclusion. The SLTA represents an effective and low-risk treatment of $\mathrm{HH}$ and leads to a substantial saving of direct costs even after 2 years, which has to be included in the costbenefit analysis of SLTA.

Keywords Epilepsy surgery - Gelastic seizures - Pediatrics . Disconnection - Minimally invasive
$10 \mathrm{mg}$ vor Ablation, anschließend $4 \mathrm{mg}$ alle $6 \mathrm{~h}$ ) verabreicht [9]. Unmittelbar nach dem Eingriff bestanden Kopfschmerzen, Verschwommensehen im linken unteren Quadranten und Gelenkschmerzen, die sich allesamt vollständig zurückbildeten. Am 10. postoperativen Tag kam es einmalig zu einem generalisierten tonischklonischen Anfall von 10 min Dauer sowie zu insgesamt 3 gelastischen Anfällen innerhalb des ersten postoperativen Monats. Bei anschließend anhaltender
Anfallsfreiheit konnte die antikonvulsive Medikation ab September 2019 (10 Monate postoperativ) abgesetzt werden - ein erneutes Anfallsereignis war bis 2 Jahre nach der Laserablation (Dezember 2020) nicht zu beobachten. Auch die antihormonelle Medikation zur Therapie der Pubertas praecox konnte ausschleichend beendet werden, nachdem sich in der endokrinologischen Verlaufskontrolle ein regelrechter Befund zeigte. In der Elektroenzephalographie ließen sich keine epilepsietypischen Potenziale oder Anfallsmuster mehr nachweisen. Das hypothalamische Hamartom stellte sich MR-morphologisch deutlich größenregredient dar und umfasste in der letzten Verlaufskontrolle (05/2020) nur noch ein kleines Residuum (• Abb. 1c, d). In der neuropsychologischen Untersuchung von 08/2020 zeigte sich eine Verbesserung des Denkens und der Verarbeitungsgeschwindigkeit bei gleichbleibend guten verbalen Lernleistungen und deut- 


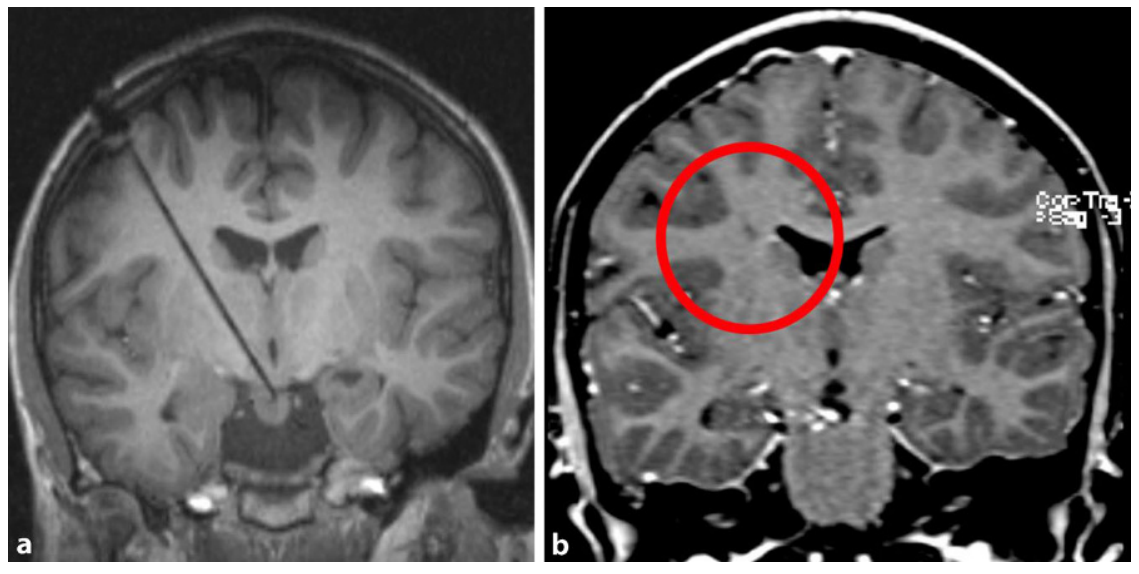

Abb. 2 ム MR-Rekonstruktion mit Darstellung der Trajektorien der Lasersonden in koronarer (a) Schnittführung. Residueller Defekte im Bereich des rechten Marklagers, dargestellt in koronarem Schnitt einer T1-Sequenz (b)

licher Verbesserung des mittelfristigen Behaltens und der Reaktionsgeschwindigkeit. Eine leichte Verschlechterung im Vergleich zur Untersuchung vor der SLTA zeigte sich lediglich im visuellen Gedächtnis. Nach der SLTA war eine normale Fortführung des Schulbesuchs möglich. Auf eine beantragte Integrationsassistenz konnte verzichtet werden. Die Wiederholung einer Klasse konnte vermieden werden, die Patientin besucht heute die 5. Klasse einer Realschule.

\section{Gesundheitsökonomische Analyse}

Zur Darstellung der Kosteneffizienz der Behandlung erfolgte eine Analyse direkter Krankheitskosten anhand der Krankenversicherungsdaten der Patientin über die Versicherungsjahre 2017 bis 2020; diese sind für die einzelnen Kostenkomponenten der $\bullet$ Abb. 3 zu entnehmen. Die gesamten direkten Kosten betrugen $€ 6603$ in 2017 sowie $€ 12.903$ in 2018 und gingen nach der Laserablation auf $€ 3609$ in 2019 und $€ 617$ in 2020 zurück. Dies entsprach im Vergleich der Jahre 2018 und 2020 einem Rückgang um $95 \%$. Die Kosten für die in den USA durchgeführte Laserablation betrugen über $€ 100.000$ und sind nicht in die Kostenrechnung eingegangen. Eine Anschlussheilbehandlung oder ambulante Rehabilitationsleistungen, wie sie nach offenem neurochirurgischem Eingriff üblich wären, waren nach der durchgeführten SLTA nicht notwendig.
Die Arbeitgeberbruttokosten für eine Integrationsassistenz belaufen sich monatlich auf etwa $€ 3000$. Unter der Annahme einer halben hier anzusetzenden Stelle also auf $€ 18.000$,-/Jahr. Nach der SLTA war bei Anfallsfreiheit eine normale Beschulung ohne die bereits beantragte Integrationsassistenz, Rollstuhl und Fahrdienst wieder möglich.

\section{Diskussion}

Die SLTA zählt an vielen Zentren, insbesondere in Nordamerika, seit mehreren Jahren als therapeutisches Procedere der ersten Wahl zur Behandlung eines symptomatischen $\mathrm{HH}[9,15]$. Eine detaillierte Beschreibung des in Deutschland zunehmend an Bekanntheit gewinnenden Verfahrens bieten die Artikel von Büntjen und Ilse et al. (2017) $[16,17]$. Demgegenüber galt über lange Zeit die mikrochirurgische Resektion als Goldstandard, barg allerdings trotz der bis zu $90 \%$ igen Chance auf Anfallsfreiheit ein ungünstiges Komplikationsrisiko von bis zu 50\% [7]. Die Komplikationen waren zumeist verursacht durch eine Schädigung der im Bereich des Zugangsweg gelegenen Strukturen wie des Fornix und der Corpora mamillaria mit Gedächtnisstörung, Läsionen des N. oculomotorius oder Thalamusinfarkten aufgrund von Verletzungen suprasellärer/interpedunkulärer Gefäße [8]. Darüber hinaus besteht durch die Nähe des HH zum Tuber cinereum ein erhöhtes Risiko für hypothalamisch/ hypophysäre Insuffizienzen mit Diabe- tes insipidus, Adipositas, Hypothyreose oder Thermoregulationsstörungen [8]. Um die Komplikationsrate zu verringern, wurden unterschiedliche Zugangswege (pterional, transventrikulär, transkallosal) und endoskopische Verfahren entwickelt, worunter eine durchgreifende Reduktion des Komplikationsrisikos allerdings nicht gelang $[8,18]$.

Eine minimal-invasive Alternative stellt die radiochirurgische Behandlung mittels Gamma-Knife dar. Eine Freiheit von behindernden Anfällen (Engel I) wurde in einer prospektiven Studie an 48 Patienten in 39,6\% (Follow-up: 36 bis 153 Monate) erzielt [19]. Besonders vorteilhaft konnten im Vergleich zur offenen operativen Therapie nach einer radiochirurgischen Therapie keine permanenten neurologischen Defizite oder Gedächtnisstörungen nachgewiesen werden [19]. Vorübergehend wurde allerdings eine transiente Zunahme der Anfallshäufigkeit bei 8 Patienten (16,6\%) beobachtet [19]. Allerdings tritt der therapeutische Effekt der Radiochirurgie zumeist erst mit einer Latenz von mehreren Wochen bis Monaten ein, was bei hochaktiven Epilepsien wie bei der vorgestellten Patientin nachteilig ist und deshalb nicht favorisiert wurde [19]. Eine Sonderform stellt die interstitielle Radiochirurgie (stereotaktische Brachytherapie) dar, bei der Implantate aus ${ }^{125} \mathrm{I}$ in das HH platziert werden. Schulze-Bonhage et al. konnten bei einem Kollektiv von 24 Patienten nach 2 Jahren in 11 Fällen eine Anfallsfreiheit oder Anfallsreduktion $>90 \%$ beobachten [20]. Das Verfahren erwies sich im Vergleich zur offenen Resektion als gut verträglich. Bei 5 Patienten entwickelte sich ein transientes Hirnödem, welches durch vorübergehende Kopfschmerzen und Fatigue symptomatisch wurde. Eine anhaltende Störung des Kurzzeitgedächtnisses fand sich in 2 Fällen, eine relevante Gewichtszunahme in 4 Fällen [20]. Eine Einschränkung des verbalen Gedächtnisses konnte in einer Studie von Wagner et al. allerdings in bis zu $50 \%$ der Fälle auch noch nach über 1 Jahr nachgewiesen werden [21]. Darüber hinaus steht dem insgesamt relativ geringen unmittelbaren Komplikationsrisiko radiochirurgischer Verfahren ein 


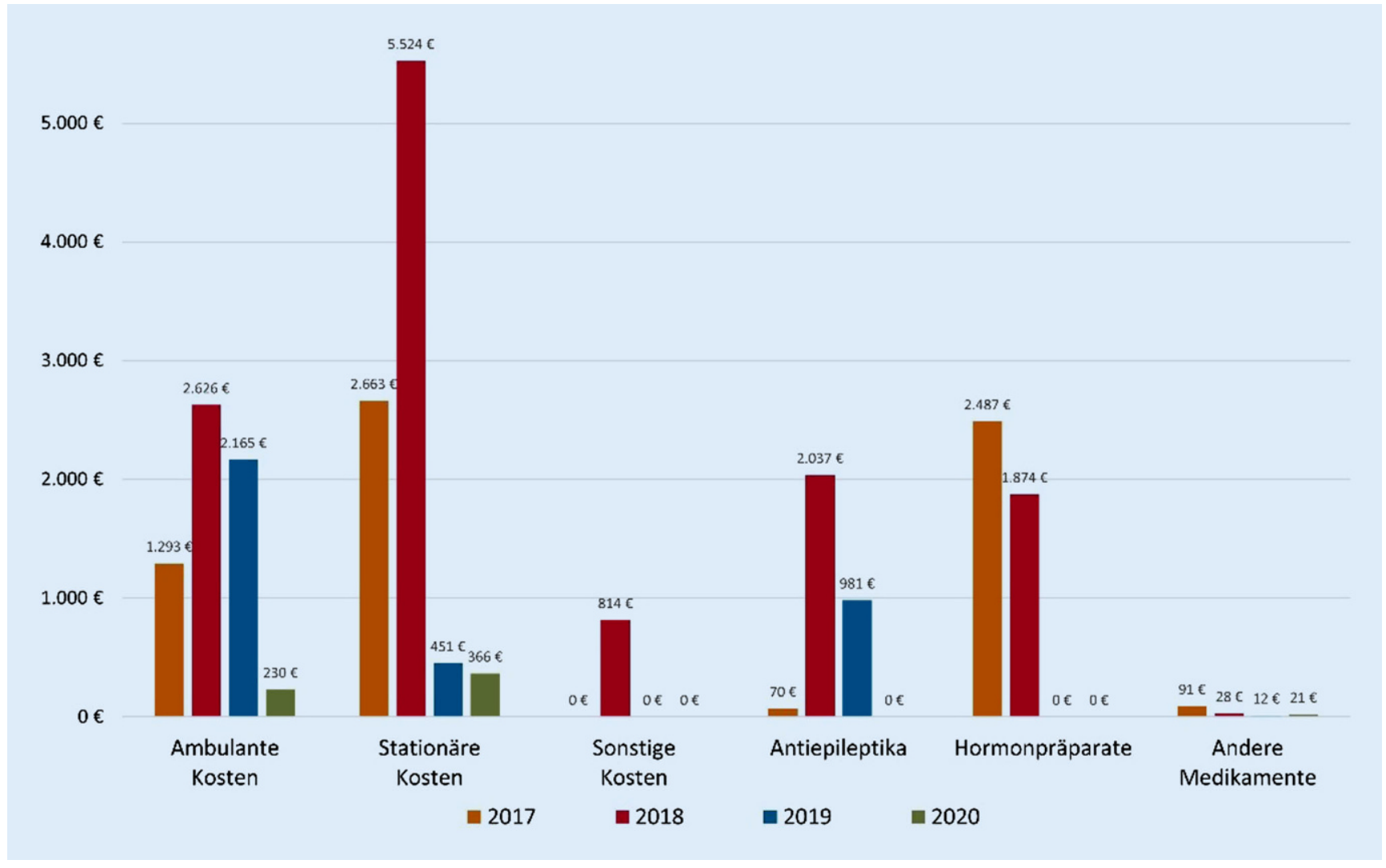

Abb. $3 \Delta$ Darstellung direkter Kosten für die Versicherungsjahre 2017 bis 2020 mit Entfallen der Kosten für Antiepileptika in 2020 und für Hormonpräparate in 2019 bei gleichzeitigem Rückgang der Kosten für stationäre und ambulante Behandlungen in 2020 und somit im 2. Jahr nach Laserablation. Die Kosten für die Laserablation in 11/2018 und die Kosten für die beantragte, aber nun nicht mehr nötige, Integrationsassistenz in Höhe von geschätzten $18.000 € / J a h r$ sind nicht dargestellt

potenziell erhöhtes Risiko für sekundäre intrakranielle Neoplasien gegenüber [22]. Zwar legen kürzlich veröffentlichte Studien bei fokalen und einzeitigen Bestrahlungen ein vergleichbares Risiko zur Normalbevölkerung nahe, es fehlen aber weiterhin aussagekräftige Langzeitdaten für Kinder, Jugendliche und junge Erwachsene, welche die Hauptzielgruppe bei der Behandlung von $\mathrm{HH}$ darstellt [23]. Insgesamt konnten sich radiochirurgische Verfahren aufgrund des verzögerten Ansprechens und des Komplikationsrisikos in der Behandlung von hypothalamischen Hamartomen nicht durchsetzen und wurden weitgehend von minimal-invasiven thermoablativen wie der SLTA oder der Radiofrequenzablation (RFA) verdrängt, zumal erschwerend hinzukommt, dass die bisherigen Daten ausschließlich aus Single-Center-Studies stammen und aussagekräftige Langzeituntersuchungen bisweilen fehlen.

Die SLTA bietet hierbei die Möglichkeit einer intraoperativen MR-Ther- mometrie, durch die eine Abschätzung der Ablationszone zur Therapieoptimierung und zum Schutz benachbarter Risikostrukturen möglich ist. In den Anfangsjahren lag die Komplikationsrate mit bis zu 23,5\% zwar noch auf einem mit der offenen Chirurgie vergleichbaren $\mathrm{Ni}$ veau, konnte mit zunehmender Expertise in spezialisierten Zentren aber auf bis zu 5,4\% reduziert werden, was den Aspekt der "Lernkurve“ und die Abhängigkeit der Komplikationsrate von der Erfahrung der neurochirurgischen Behandler hervorhebt [9, 24]. Die Anfallsfreiheit nach 1 Jahr lag in einer Studie von Curry et al. (2018) für gelastische Anfälle bei $93 \%$ und zeigte somit ein vergleichbares Ansprechen wie nach offener Resektion, wenngleich ein Engel-IA-Outcome (komplette Anfallsfreiheit) ohne Medikation nur in $12 \%$ zu erzielen war [9]. Ähnliche Ergebnisse wurden mittels stereotaktischer Radiofrequenzablation veröffentlicht: Eine umfangreiche Studie der Nishi-Niigata-Gruppe aus Japan berichtete in einem Kollektiv von 100 Patienten mit einem medianen Followup von 3 Jahren („range“: 1 bis 17 Jahre) von einer Anfallsfreiheit für gelastische Anfälle in $86 \%$ und einer kompletten Anfallsfreiheit in 71\% [25]. Der höheren Rate an kompletter Anfallsfreiheit standen allerdings ein deutlich höherer Anteil transienter Komplikationen (60\% Horner-Syndrom, 27,9\% Hyperphagie, 22,1\% Hyponatriämie, 22,1\% Hyperthermie, 8,6\% Kurzzeitgedächtnisstörung, 2,9\% intrakranielle Blutung) sowie einer permanenten hypophysären Insuffizienz (2 Patienten; 2\%) gegenüber; zusätzlich berichteten Curry et al. im Vergleich zur Nishi-Niigata Gruppe eine geringere Rate an Re-Operationen (23\% vs. $32 \%)[9,25]$.

Während zu Beginn die Auffassung vertreten wurde, dass eine vollständige Resektion oder Ablation des HH notwendig sei, um eine Anfallsfreiheit zu erzielen, stellte sich mit der Zeit heraus, dass zumeist nur ein Teil des HH die 
eigentliche epileptogene Zone darstellt und entweder eine gezielte Zerstörung oder Diskonnektion vom epileptischen Netzwerk ausreichend ist [12]. Bourdillon et al. (2020) berichteten in einem rezenten Review, dass durch die kleineren Ablationszonen der stereotaktischen RFA zwar eine zielgenauere Ablation des gesamten $\mathrm{HH}$ möglich sei, eine initialvermutete Überlegenheit der RFA hinsichtlich der Anfallsfreiheit allerdings durch die zunehmende Erfahrung mit selektiven Ablationen mittels SLTA und dem Konzept der Diskonnektion bei gleichzeitig günstigerem Komplikationsrisiko relativiert wird [7]. Eine Möglichkeit zur Determinierung der epileptogenen Zone stellt eine funktionelle MRT zur Messung der „resting state connectivity“ (rsfMRI) dar, welche ebenfalls präoperativ bei der vorgestellten Patientin durchgeführt wurde [14]. Die Studie von Boerwinkle et al. zeigte mit einem Engel-IOutcome bei $91,7 \%$ gegenüber $46,7 \%$ ohne rs-fMRI ein signifikant besseres therapeutisches Ergebnis. Durch das genaue Mapping war eine Schonung der $\mathrm{HH}$ Übergangszone möglich, was sich in einer Komplikationsrate von $0 \%$ widerspiegelte [14]. In • Abb. 2 sind die Trajektorien $\mathrm{zu}$ erkennen, welche in dem $\mathrm{HH}$ mündeten. In der Verlaufskontrolle ist ein asymptomatischer und prognostisch irrelevanter Gewebedefekt entlang des Zugangswegs zu erkennen. Da das abladierte Gewebe im Vergleich zur Resektion nicht sofort entfernt wird, kann es insbesondere noch in den ersten Tagen und Wochen zu Anfallsrezidiven kommen, bis die Ablationszone durch körpereigene Prozesse abgebaut wurde. Eine suffiziente Einschätzung des Therapieerfolgs kann daher zumeist erst nach 6 bis 12 Monaten erfolgen [26]. Darüber hinaus kann es auch im weiteren Verlauf zu einer Abnahme der Anfallshäufigkeit und epileptischer Aktivität im EEG im Sinne eines Running-down-Phänomens kommen, bei dem angenommen wird, dass durch die Ablation der primären epileptogenen Zone eines epileptischen Netzwerkes sekundäre Bereiche stumm geschaltet werden [27]. Da dieser Effekt mit der Dauer der Epilepsie und der Ausbildung robuster epileptischer Netzwerke geringer werden kann, ist eine frühzeitige
Behandlung des $\mathrm{HH}$ von entscheidender Bedeutung, um die Entstehung extrahypothalamischer epileptogener Zonen zu verhindern [28].

Neben einer strukturellen Epilepsie können $\mathrm{HH}$ endokrinologische Erkrankungen wie eine Pubertas praecox verursachen [29]. Pathophysiologisch wird eine Stimulation der Gonadotropin-releasing-hormon(GnRH)-Freisetzung durch das $\mathrm{HH}$ vermutet. Da die hormonelle Stimulation durch Resektion des $\mathrm{HH}$ wegfällt, kann im Anschluss an die Resektion zumeist auch eine rasche Normalisierung des Hormonhaushalts erreicht werden [30]. Im Falle einer isolierten Pubertas praecox wird als primäre Therapie die verhältnismäßig nebenwirkungsarme Gabe von GnRH-Antagonisten empfohlen [29].

Während die SLTA in den Vereinigten Staaten bereits in 2011 eingeführt wurde, erfolgte eine CE-Zertifizierung in Europa erst in 2018. Die erste SLTA in Deutschland wurde im März 2019 am Universitätsklinikum Magdeburg bei einem Erwachsenen mit therapierefraktärer Temporallappenepilepsie durch SLTA des rechtsseitigen Amygdala-Hippocampus-Komplexes durchgeführt [31]. Eine breite Anwendung in Deutschland besteht aktuell nicht, da eine kostendeckende Durchführung der Prozedur bislang im DRG-System nicht abgebildet ist.

Berechnungen zur kostendeckenden Durchführung der Prozedur ergaben für das Universitätsklinikum Frankfurt stationäre Behandlungskosten einschließlich des Materialverbrauches von ca. $60.000 €$ pro Prozedur. Diese Kosten sind insbesondere dem erwarteten Nutzen bei zu erreichender Anfallsfreiheit gegenüberzusetzen. In dem berichteten Fall konnten die direkten Krankheitskosten von ca. $€ 12.000$,- auf unter $€ 1000$,-/ Jahr gesenkt werden. Dies deckt sich mit publizierten gesundheitsökonomischen Berechnungen, die sehr hohe direkte Kosten beim therapierefraktären Verlauf aufzeigen [5, 32, 33]. Bei Kindern und Jugendlichen sind beim therapierefraktären Verlauf jährliche direkte Kosten in Höhe von $13.850 €$ und indirekte Kosten in Höhe von $10.760 €$ für den Arbeitsausfall der Eltern zu erwarten [5]. Bei Erwachsenen mit therapierefraktären
Anfällen betragen die direkten Kosten $8250 €$ und die indirekten Kosten $9170 €$ pro Jahr, für Letzteres sind Arbeitslosigkeit, Teilzeitarbeit, Fehltage und Frühberentung aufgrund der Epilepsie zu nennen [34]. Nach erfolgreicher Epilepsiechirurgie nehmen diese Kosten in der Regel nach 2 bis 3 Jahren ab [35]. Bei dem geschilderten Fall sind auch Kosten für bereits beantragte Maßnahmen und Hilfsmittel aufgezeigt worden, die sich auf geschätzte $€ 18.000$,-/Jahr allein für die Integrationsassistenz beliefen. Diese werden in Krankheitskostenstudien teilweise nicht berücksichtigt. Darüber hinaus sollten stets auch indirekte Kosten in Betracht gezogen werden, die durch den Arbeitsausfall sowohl bei den Patienten wie bei Eltern oder Betreuenden der Betroffenen entstehen können [5, 32, 33]. Schwer zu bemessen, aber an diesem Fall nachvollziehbar, sind die hohen intangiblen Kosten für das betroffene Kind und die Eltern, die nach der erfolgreichen SLTA nicht mehr fortbestanden.

\section{Schlussfolgerung}

Die stereotaktische Laserthermoablation (SLTA) stellt eine effiziente und relativ komplikationsarme Therapie zur Behandlung eines symptomatischen hypothalamischen Hamartoms dar, durch die eine Anfallsfreiheit und Normalisierung endokrinologischer Störungen erzielt werden kann. Dadurch können jährliche direkte Kosten für die Krankenkassen effektiv gesenkt werden, was den Behandlungskosten einer SLTA gegenübergestellt werden sollte.

\section{Korrespondenzadresse}

\section{Dr. med. Konstantin Kohlhase}

Klinik für Neurologie und Epilepsiezentrum Frankfurt Rhein-Main, Goethe-Universität Frankfurt

Schleusenweg 2-16, 60528 Frankfurt am Main, Deutschland

Konstantin.kohlhase@kgu.de

Funding. Open Access funding enabled and organized by Projekt DEAL. 


\section{Einhaltung ethischer Richtlinien}

Interessenkonflikt. Das Universitätsklinikum Frankfurt, die BIG Krankenkasse und die Fa. Medtronic haben zusammen einen trilateralen Vertrag nach $\S 140$ a SGB V über die Erbringung der besonderen Versorgung stereotaktische Laserthermoablation bei Patienten mit Epilepsie. D.J. Curry gibt an, ein Berater für die Fa. Medtronic zu sein. K. Kohlhase, A. Strzelczyk, L. Porto, A. Beißel, F. Rosenow und S. Schubert-Bast geben an, dass keine weiteren relevanten Interessenskonflikte bestehen.

Für diesen Beitrag wurden von den Autoren keine Studien an Menschen oder Tieren durchgeführt. Die Eltern der vorgestellten Patientin gaben ihr Einverständnis zur Publikation des Fallberichtes. Für die aufgeführten Studien gelten die jeweils dort angegebenen ethischen Richtlinien.

Open Access. Dieser Artikel wird unter der Creative Commons Namensnennung 4.0 International Lizenz veröffentlicht, welche die Nutzung, Vervielfältigung, Bearbeitung, Verbreitung und Wiedergabe in jeglichem Medium und Format erlaubt, sofern Sie den/die ursprünglichen Autor(en) und die Quelle ordnungsgemäß nennen, einen Link zur Creative Commons Lizenz beifügen und angeben, ob Änderungen vorgenommen wurden.

Die in diesem Artikel enthaltenen Bilder und sonstiges Drittmaterial unterliegen ebenfalls der genannten Creative Commons Lizenz, sofern sich aus der Abbildungslegende nichts anderes ergibt. Sofern das betreffende Material nicht unter der genannten Creative Commons Lizenz steht und die betreffende Handlung nicht nach gesetzlichen Vorschriften erlaubt ist, ist für die oben aufgeführten Weiterverwendungen des Materials die Einwilligung des jeweiligen Rechteinhabers einzuholen.

Weitere Details zur Lizenz entnehmen Sie bitte der Lizenzinformation auf http://creativecommons.org/ licenses/by/4.0/deed.de.

\section{Literatur}

1. Sperling MR, Barshow S, Nei M, Asadi-Pooya AA (2016) A reappraisal of mortality after epilepsy surgery. Neurology 86:1938-1944

2. Strzelczyk A, Griebel C, Lux W, Rosenow F, Reese JP (2017) The burden of severely drug-refractory epilepsy: a comparative longitudinal evaluation of mortality, morbidity, resource use, and cost using German health insurance data. Front Neurol 8:712

3. Harvey AS, Freeman JL (2007) Epilepsy in hypothalamic hamartoma: clinical and EEG features. Semin Pediatr Neurol 14(2):60-64

4. Corbet Burcher G, Liang H, Lancaster R et al (2019) Neuropsychiatric profile of paediatric hypothalamichamartoma: systematic review and case series. Dev Med Child Neurol 61(12):1377-1385

5. Riechmann J, Strzelczyk A, Reese JP et al (2015) Costs of epilepsy and cost-driving factors in children, adolescents, and their caregivers in Germany. Epilepsia 56(9):1388-1397

6. Riechmann J, Willems LM, Boor R et al (2019) Quality of life and correlating factors in children, adolescents with epilepsy, and their caregivers: a cross-sectional multicenter study from Germany. Seizure 69:92-98
7. Bourdillon P, Ferrand-Sorbet S, Apra C et al (2020) Surgical treatment of hypothalamic hamartomas. Neurosurg Rev 44(2): 753-762

8. Mittal S, Mittal M, Montes JL, Farmer JP, Andermann F (2013) Hypothalamic hamartomas. Part 2. Surgical considerations and outcome. Neurosurg Focus 34(6):1-10

9. Curry DJ, Raskin J, Ali I, Wilfong AA (2018) MR-guided laser ablation for the treatment of hypothalamic hamartomas. Epilepsy Res 142:131-134

10. Kohlhase K, Zöllner JP, Tandon N, Strzelczyk A, Rosenow $F$ (2021) Comparison of minimally invasive and traditional surgical approaches for refractory mesial temporal lobe epilepsy: a systematic review and meta-analysis of outcomes. Epilepsia 62(4):831-845

11. Strzelczyk A, Reese JP, Dodel R, Hamer HM (2008) Cost of epilepsy: a systematic review. PharmacoEconomics 26(6):463-476

12. Delalande O, Fohlen M (2003) Disconnecting surgical treatment of hypothalamic hamartoma in children and adults with refractory epilepsy and proposal of a new classification. Neurol Med Chir 43:61-68

13. Rosenow F, Bast T, Czech T et al (2016) Revised version of quality guidelines for presurgical epilepsy evaluation and surgical epilepsy therapy issued by the Austrian, German, and Swiss working group on presurgical epilepsy diagnosis and operative epilepsy treatment. Epilepsia 57(8):1215-1220

14. Boerwinkle VL, Foldes ST, Torrisi SJ et al (2018) Subcentimeter epilepsy surgery targets by resting state functional magnetic resonance imaging can improve outcomes in hypothalamic hamartoma. Epilepsia 59(12):2284-2295

15. DuVX,GandhiSV, RekateHL,MehtaAD (2017) Laser interstitial thermal therapy: A first line treatment for seizures due to hypothalamic hamartoma? Epilepsia 58(Suppl 2):77-84

16. Büntjen L, Voges J, Heinze HJ, Hinrichs $H$, Schmitt FC (2017) Stereotactic laser ablation: technical concepts und clinical application. ZEpileptol 30(2):138-145

17. Ilse A, Büntjen L, Schmitt FC (2017) Stereotaktische Laserablation für fokale Epilepsien: Eine Literaturübersicht. ZEpileptol 30(2):152-161

18. Ng YT, Rekate HL, Prenger EC et al (2008) Endoscopic resection of hypothalamic hamartomas for refractory symptomatic epilepsy. Neurology 70(17):1543-1548

19. Régis J, Lagmari M, Carron R et al (2017) Safety and efficacy of gamma knife radiosurgery in hypothalamic hamartomas with severe epilepsies: a prospective trial in 48 patients and review of the literature. Epilepsia 58(Suppl 2):60-71

20. Schulze-Bonhage A, Trippel $M$, Wagner $K$ et al (2008) Outcome and predictors of interstitial radiosurgery in the treatment of gelastic epilepsy. Neurology 71(4):277-282

21. Wagner K, Buschmann F, Zentner J, Trippel M, Schulze-Bonhage A (2014) Memory outcome one year after stereotactic interstitial radiosurgery in patients with epilepsy due to hypothalamic hamartomas. Epilepsy Behav 37:204-209

22. Braunstein S, Nakamura JL (2013) Radiotherapyinduced malignancies: review of clinical features, pathobiology, and evolving approaches for mitigating risk. Front Oncol 3:73

23. Wolf A, NaylorK, Tam Metal (2019) Risk ofradiationassociated intracranial malignancy after stereotactic radiosurgery: a retrospective, multicentre, cohort study. Lancet Oncol 20(1):159-164
24. Hoppe C, WittJA, HelmstaedterC, GasserT, VatterH, Elger CE (2017) Laser interstitial thermotherapy (LITT) in epilepsy surgery. Seizure 48:45-52

25. Kameyama S, Shirozu $H$, Masuda $H$, Ito $Y$, Sonoda M, Akazawa K (2016) MRI-guided stereotactic radiofrequency thermocoagulation for 100 hypothalamic hamartomas. J Neurosurg 124(5):1503-1512

26. Xu DS, Chen T, Hlubek RJ et al (2018) Magnetic resonance imaging-guided laser interstitial thermal therapy for the treatment of hypothalamic hamartomas: a retrospective review. Clin Neurosurg 83(6):1183-1191

27. Yang C, Liu Z, Luan G, Wang Q (2020) The extension of epileptogenicity as the driving force of the epileptogenic network evolution and complex symptoms. Brain Res 1748:147073

28. Scholly J, Valenti MP, Staack AM et al (2013) Hypothalamic hamartoma: Is the epileptogenic zone always hypothalamic? Arguments for independent (third stage) secondary epileptogenesis. Epilepsia 54(Suppl 9):123-128

29. Harrison VS, Oatman O, Kerrigan JF (2017) Hypothalamichamartoma with epilepsy: review of endocrine comorbidity. Epilepsia 58(2):50-59

30. Luo S, Li C, Ma Z, Zhang Y, Jia G, Cheng Y (2002) Microsurgical treatment for hypothalamic hamartoma in children with precocious puberty. Surg Neurol 57(5):356-362

31. Schmitt FC, Büntjen L, Schütze $H$ et al (2020) Stereotactic laser thermal ablation of mesial temporal lobe epilepsy with right hippocampal sclerosis-patient decision-making, realization and visualization of memory function. Z Epileptol 33(1):42-49

32. Willems LM, Richter S, Watermann $N$ et al (2018) Trends in resource utilization and prescription of anticonvulsants for patients with active epilepsy in Germany from 2003 to 2013-a ten-year overview. Epilepsy Behav 83:28-35

33. Strzelczyk A, Nickolay T, Bauer S et al (2012) Evaluation of health-care utilization among adult patients with epilepsy in Germany. Epilepsy Behav 23(4):451-457

34. Strzelczyk A, Reese JP, Oertel WH, Dodel R, Rosenow F, Hamer HM (2013) Costs of epilepsy and their predictors: cross-sectional study in Germany and review of literature. Epileptology 1:55-60

35. Picot MC, Jaussent A, Neveu D et al (2016) Cost-effectiveness analysis of epilepsy surgery in a controlled cohort of adult patients with intractable partial epilepsy: a 5-year follow-up study. Epilepsia 57(10):1669-1679 